\title{
Auger recombination dynamics of lead salts under picosecond free-electron-laser excitation
}

\author{
P. C. Findlay and C. R. Pidgeon \\ Physics Department, Heriot Watt University, Edinburgh EH14 4AS, United Kingdom \\ R. Kotitschke, A. Hollingworth, and B. N. Murdin \\ Physics Department, University of Surrey, Guildford GU2 5XH, United Kingdom \\ C. J. G. M. Langerak and A. F. G. van der Meer \\ FOM Institute for Plasma Physics, “Rijnhuizen,'” P. O. Box 1207, NL-3430 BE Nieuwegein, The Netherlands \\ C. M. Ciesla \\ Toshiba Cambridge Research Center Ltd., 260 Cambridge Science Park, Cambridge CB4 4WE, United Kingdom \\ J. Oswald and A. Homer \\ Institut für Physik der Montanuniversität Leoben, Franz, Josef Straße 18, A-8700 Leoben, Austria \\ G. Springholz and G. Bauer \\ Semiconductor Physics Group, Universität Linz, A-4040 Linz, Austria
}

(Received 11 May 1998)

\begin{abstract}
Pump-probe transmission experiments have been performed on $\mathrm{PbSe}$ above the fundamental absorption edge near $4 \mu \mathrm{m}$ in the temperature range 30 to $300 \mathrm{~K}$, using the Dutch ps free-electron laser. For temperatures below $200 \mathrm{~K}$ and carrier densities above the threshold for stimulated emission, stimulated recombination represents the most efficient recombination mechanism with relatively fast kinetics in the 50-100-ps regime, in good agreement with earlier reports of photoluminescent emission. Above this temperature Auger recombination dominates, and the Auger coefficient $C$ is determined from the pump-probe decay curves. In the lowtemperature regime the Auger coefficient is determined from the decay curves at times beyond 100 ps. The Auger coefficient is approximately constant (with a value of about $8 \times 10^{-28} \mathrm{~cm}^{6} \mathrm{~s}^{-1}$ ) between 300 and $70 \mathrm{~K}$, and then drops a value of about $1 \times 10^{-28} \mathrm{~cm}^{6} \mathrm{~s}^{-1}$ at $30 \mathrm{~K}$, in good agreement with the theory for nonparabolic near-mirror bands and nondegenerate statistics. It is found that $C$ for PbSe is between one and two orders of magnitude lower than for $\mathrm{Hg}_{1-x} \mathrm{Cd}_{x} \mathrm{Te}$ of comparable band gap. [S0163-1829(98)07243-9]
\end{abstract}

\section{INTRODUCTION}

Lead salts are of interest both fundamentally, because of their interesting band structure, and from a device point of view since they are widely used in midinfrared (MIR) optoelectronic emitters and detectors. A particular feature of this system-i.e., their near "mirror" conduction and valence bands - has been cited as potentially leading to much smaller Auger scattering rates than found in III-V material systems. However, theory predicts that there may nevertheless still remain a substantial contribution to this unwanted recombination process through intervalley scattering, ${ }^{1-4}$ although later theories, ${ }^{2-4}$ including more realistic band-structure pictures, suggest that this should not be as serious as originally predicted. ${ }^{1}$ Understanding accurately how the recombination processes change with carrier density is important for emission devices which operate far from equilibrium, and in particular in the continuing quest for the development of compact MIR room-temperature lasers. A recent design study suggesting the possibility of $\mathrm{cw}$ room-temperature MIR operation of $\mathrm{GaSb} / \mathrm{PbSe} / \mathrm{GaSb}$ double heterostructure lasers ${ }^{5}$ depends critically on the theoretical prediction of a low value of Auger coefficient $C{ }^{4}$ Although considerable research has been done on the recombination processes, with the excep- tion of one report ${ }^{6}$ almost all previous work has utilized techniques using relatively long pulse or $\mathrm{cw}$ radiation, and experimentally reported values for $C$ vary over an order of magnitude. ${ }^{6-8}$

We have made direct pump-probe measurements of radiative and nonradiative recombination in epilayers of $\mathrm{PbSe}$ under high excitation with an rf linac-pumped (ps) free-electron laser (FELIX), in the temperature range 30-300 K. For temperatures below $200 \mathrm{~K}$ and carrier densities above the threshold for stimulated emission, stimulated recombination represents the most efficient recombination mechanism with kinetics in the 50-100-ps regime, in good agreement with earlier reports. ${ }^{6,9,10}$ Above this temperature Auger recombination dominates on a $0.1-2$-ns time scale. The sophistication of a three-beam pump-probe experimental technique described below has enabled an analysis of the decay at times longer than $100 \mathrm{ps}$ in terms of the nonparabolic anisotropic band structure of the lead salts, to give the Auger coefficient $C$ qualitatively over a wide temperature range $30-300 \mathrm{~K}$. The results are in good agreement with theory for nonparabolic, anisotropic bands ${ }^{11,12}$ and nondegenerate statistics, ${ }^{2}$ and confirm that the original parabolic band calculations ${ }^{1}$ underestimated the measured lifetime by more than an order of magnitude. ${ }^{2-4,6}$ Finally the Auger results for the lead salts 


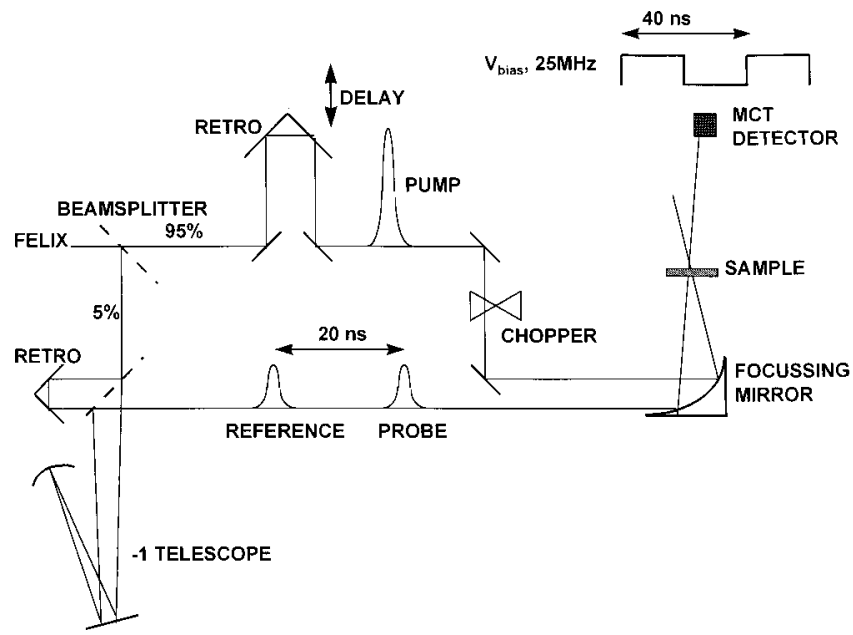

FIG. 1. Schematic diagram of the three-beam pump-probe experimental setup, utilizing a modulation technique to determine the relative transmission of the probe beam as a function of delay time as described in the text.

are compared with earlier measurements taken at FELIX (Ref. 13) for $\mathrm{Hg}_{1-x} \mathrm{Cd}_{x} \mathrm{Te}$ of a composition such that the band gap for the two semiconductors is the same at $T=100$ $\mathrm{K}{ }^{13}$ It is found that $C$ for $\mathrm{PbSe}$ is between one and two orders of magnitude lower than for $\mathrm{Hg}_{1-x} \mathrm{Cd}_{x}$ Te over the whole temperature range for comparable values of the band gap. This results in domination of stimulated radiative recombination even at quite low carrier concentrations.

\section{EXPERIMENT}

The samples were (111)-oriented epitaxial layers of PbSe on insulating substrates $\left(\mathrm{BaF}_{2}\right)$. They were grown by molecular-beam epitaxy under ultrahigh-vacuum conditions. ${ }^{14}$ The samples were $\sim 2 \mu \mathrm{m}$ thick with an $n$-type carrier concentration of $3.2 \times 10^{17} \mathrm{~cm}^{-3}$ and a mobility of $28070 \mathrm{~cm}^{2} \mathrm{~V}^{-1} \mathrm{~s}^{-1}$ at $77 \mathrm{~K}$. The wavelength-degenerate pump-probe experiments were performed with the Dutch free electron laser in Utrecht (FELIX), which operated with pulse trains ("macropulses") typically of length $4 \mu$ s and at a repetition rate of $5 \mathrm{~Hz}$. The macropulse consisted of a train of "micropulses," each of width adjustable between 2 and $10 \mathrm{ps}$, with a pulse separation of $40 \mathrm{~ns}$. It was shown previously $^{13,15}$ that, over the range of excited electron concentrations used throughout this study, the carrier temperatures approach the lattice temperature to within $2 \%$ in less than $3 \mathrm{ps}$, resulting in a temperature increase in the sample of only $\sim 0.02 \mathrm{~K}$ per pulse. Hence we have neglected heating effects and, for recombination processes which occur in less than about $20 \mathrm{~ns}$ (see below), we have treated the interpretation in the same way as for a "single pulse" experiment.

The macropulse fluctuations of FELIX depend strongly on the required performance of the machine, and can be of order $10 \%$. In order to obtain a good signal-to-noise ratio in the MIR regime, we have utilized a pump-probe setup which compensates for these macropulse fluctuations. The experimental arrangement is a three-beam pump-probe-reference setup shown in Fig. 1. From the original pump pulse both a probe beam and a reference beam are split off by $\mathrm{ZnSe}$ beamsplitters. The reference beam is delayed by $20 \mathrm{~ns}(6 \mathrm{~m})$, and back-reflected on the beamsplitter with a -1 telescope onto the probe beam position. The probe and reference beams follow the same optical path, transmit through the sample at the same position, and are both detected by the same $(77 \mathrm{~K}) \mathrm{MCT}$ detector. It is assured independently that both have the same original size. The detector bias is modulated with $25 \mathrm{MHz}$, synchronized with FELIX. This results in signals with opposite sign for the probe and the reference beams. When the system is in balance (no pump is applied), the integrating electronics show an apparent output signal of the detector which is zero. In the experiment the pump beam is chopped with $2.5-\mathrm{Hz}$ frequency (every other pump pulse is blocked) and the signal fed into a boxcar integrator in the toggle mode. The result is a signal-to-noise ratio better than $0.1 \%$, even when the fluctuations from macropulse to macropulse are several percent. The three beams were focused on the sample using an $f=25 \mathrm{~cm}$ parabolic mirror, resulting in a spotsize of $100 \mu \mathrm{m}$. The sample was mounted in a flow cryostat $(4-300 \mathrm{~K})$. The effective pump and probe energy fluences per micropulse were estimated to be 350 and 10 $\mu \mathrm{J} / \mathrm{cm}^{2}$, respectively, including losses due to beamsplitters and optics. The relative transmittance of the probe was measured directly as a function of optical delay, $t_{\text {delay }}$, between pump and probe pulses.

Band filling as the pump radiation is absorbed causes a strong dynamic blueshift in the IR absorption edge (the dynamic Moss-Burstein shift), and leads to pronounced bleaching near the excitation frequency. Recovery times in the range $20-800$ ps were found, and shown to be strongly dependent on the sample temperature and the excited carrier density. In agreement with previous workers, ${ }^{6}$ we have found that at room temperature and at the pump intensities (excited carrier densities) used here the recombination is dominated by Auger scattering as shown in Fig. 2 (and see the analysis below). Below $200 \mathrm{~K}$, stimulated recombination represents the most efficient recombination mechanism with kinetics in the 100-ps regime, as shown in Fig. 3. At times longer than $100 \mathrm{ps}$, the Auger process takes over in the absence of stimulated recombination. For all temperatures the net recombination rate in the high excitation regime is a fundamental property of the material, and not defect related. ${ }^{13,15}$ Analysis of the recovery of the probe absorption leads to a quantitative determination of the temperature dependence of the threshold concentration for stimulated emission and the Auger coefficient, as described below.

\section{ANALYSIS}

In order to interpret the data of Figs. 2 and 3, we need to convert the measured transmission into values of excited carrier concentration $N_{e}$. The rate of decay of $N_{e}(t)$ with pump-probe delay can then be extracted directly, and Auger recombination coefficients can be obtained by fitting $N_{e}(t)$ with a simple rate equation. The recovery time of the bleaching depends on the photon energy $h \nu$, due to the carrierconcentration-dependent relaxation process. The value of $N_{e}$ at complete bleaching corresponds to the carrier density required to separate the electron and hole quasi-Fermi energies by an amount equal to the pump photon energy, and increases monotonically with $h \nu$. We assume that the carriers thermalize rapidly compared with the recombination rate, in 


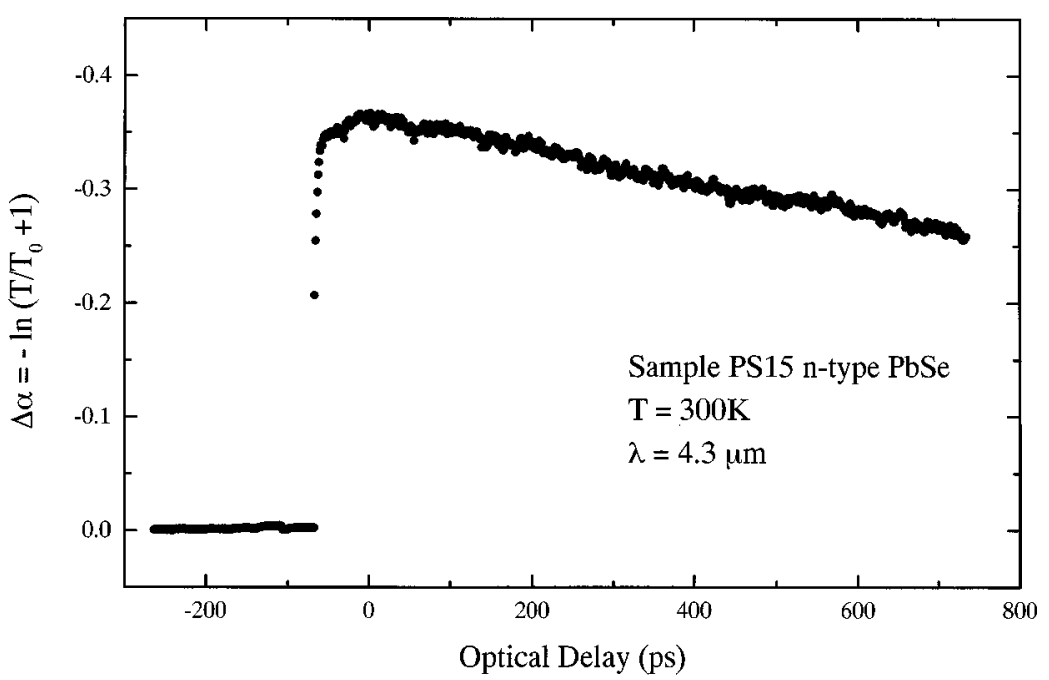

FIG. 2. Pump-probe transmission results for PbSe sample (PS15), at $T=300 \mathrm{~K}$, for a pump wavelength of $4.3 \mu \mathrm{m}$, measured directly from our modulation technique as $\Delta \alpha=-\log \left(T / T_{0}+1\right)$ vs $t_{\text {delay }}\left(T\right.$ and $T_{0}$ : transmission with and without excitation).

which case the concentration becomes determined: every value of transmission corresponds to a unique electron and hole quasi-Fermi energy and thus a unique excited carrier concentration, since the electron and hole concentrations are equal. With the measured lifetimes substantially longer than the pulse duration $(5 \mathrm{ps})$, the generation rate is zero during the probe pulse delay time, and the recombination rate $\gamma$, which is some function of $N_{e}(t)$, can be measured unambiguously.

We may similarly neglect the pulse duration [i.e., the probe pulse shape function $I(t)$ is effectively a $\delta$ function] when calculating the sample transmission.

$$
T \approx(1-R)^{2} \exp \left[-\alpha\left(t_{\text {delay }}\right) d\right] .
$$

We have assumed that the absorption coefficient $\alpha$, while time dependent, is approximately constant through the probed region of the sample (the initial concentration is spatially uniform for complete bleaching). $R$ is the sample reflectivity, and $d$ the thickness. The dependence of the transmission on the reflectivity is complicated by the different values for the top $\mathrm{PbSe}$ layer, the bottom $\mathrm{BaF}_{2}$ layer, and the interface between the epilayer and the substrate. This problem is removed in our signal processing method, where we take the ratio of the measured difference in the probe and reference transmission to the reference transmission. In this way the dependence of the reflectivity is removed, leaving us with an expression relying solely on the change in the absorption coefficient $\Delta \alpha$, resulting from the pump:

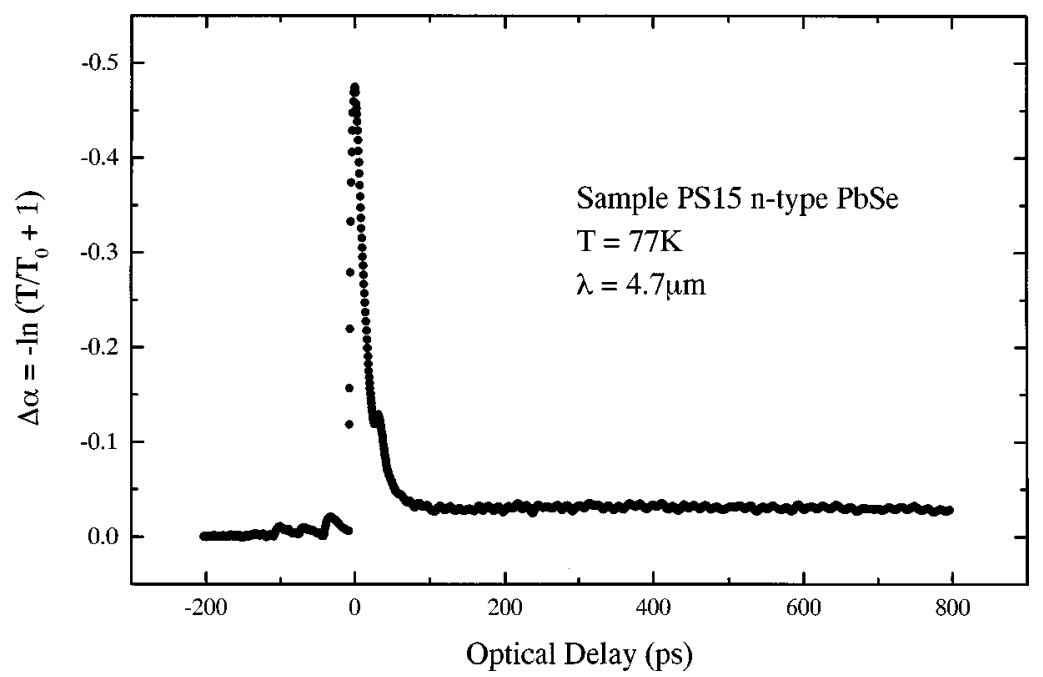

FIG. 3. Pump-probe transmission results for PbSe sample (PS15), at $T=77 \mathrm{~K}$, for a pump wavelength of $4.7 \mu \mathrm{m}$, measured directly from our modulation technique as $\Delta \alpha=-\log \left(T / T_{0}+1\right)$ vs $t_{\text {delay }}\left(T\right.$ and $T_{0}$ : transmission with and without excitation). 


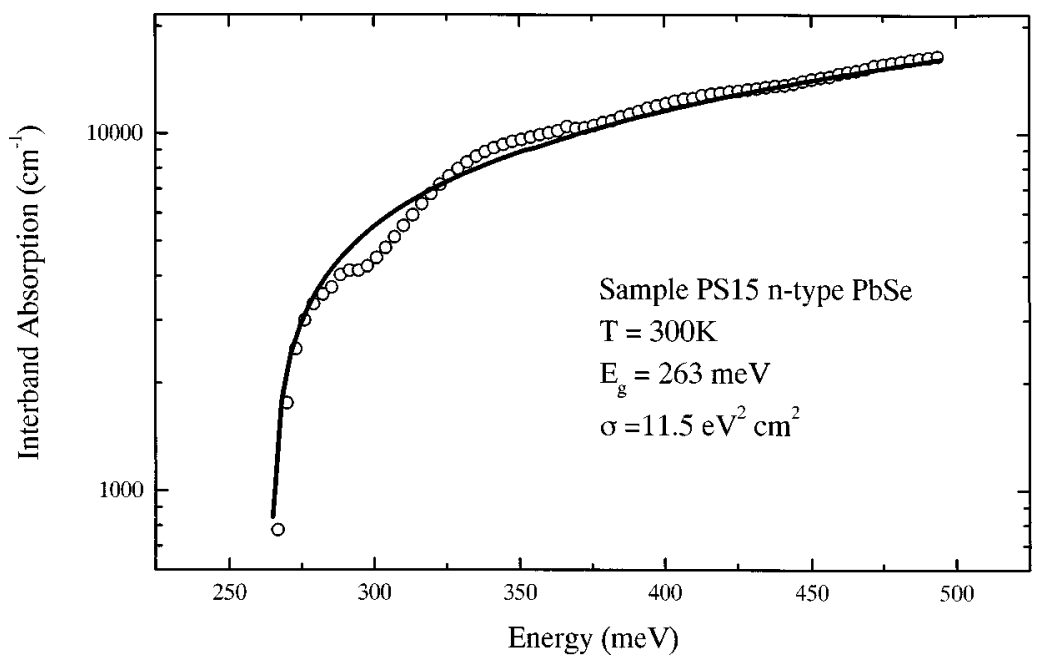

FIG. 4. Interband absorption coefficient as a function of energy for PbSe at $300 \mathrm{~K}$ in the region of the absorption edge. The experimental points (circles) were taken with a Fourier transform spectrometer. The solid line is fitted from Eqs. (3)-(7) to determine the value of the small signal absorption cross section.

$$
\frac{T_{\text {probe }}-T_{\text {reference }}}{T_{\text {reference }}}=\frac{(1-R)^{2}\left[\exp \left\{-\left(\alpha_{0}+\Delta \alpha\right) d\right\}-\exp \left(-\alpha_{0} d\right)\right]}{(1-R)^{2}\left[\exp \left(-\alpha_{0} d\right)\right]}=\exp [-(\Delta \alpha) d]-1 .
$$

The procedure is then to use a knowledge of the nonspherical, nonparabolic lead salt band structure (see below), which determines the (final and initial) electron and hole energies $E_{e}$ and $E_{h}$ for electron-hole pair creation at a given photon energy, and also the density-of-state functions $g_{e}$ and $g_{h}$. One then takes a range of values of electron Fermi energy, and computes the corresponding hole Fermi energies and electron and hole excited carrier concentrations from the relation

$$
N_{e}=\int_{E_{0}}^{\infty} f_{e}(E) g_{e}(E) d E=\int_{-\infty}^{E_{v}} f_{h}(E) g_{h}(E) d E,
$$

where $f_{e}$ and $f_{h}$ are the Fermi occupation probability functions for the electron and hole respectively, and $E_{c}$ and $E_{v}$ are the band-edge energies for the conduction and valence bands. At the same time, each assumed value of electron Fermi energy determines a unique value of $\alpha$ (and hence $T$ ) from the relation

$$
\alpha=\frac{\sigma}{h \nu}\left[1-f_{e}\left(E_{e}\right)-f_{h}\left(E_{h}\right)\right] J_{\mathrm{cv}}(E),
$$

where $J_{\mathrm{cv}}(E)$ is the joint density of states, and the absorption cross section $\sigma$ is determined by fitting the theoretical transmission obtained with the equilibrium Fermi energy to the small signal absorption spectrum taken with a Fourier transform spectrometer. This is shown in Fig. 4, utilizing Eqs. (4) -(7) (below) for the theoretical fit. We estimate the overall error in deriving the carrier concentration from the pumpprobe measurement is about $30 \%$, leading to an error in the Auger coefficient of about $60 \%$.

Intraband and interband magneto-optical experiments on $\mathrm{PbSe}$ have been interpreted previously using a k.p model which takes into account the levels forming the minimum gap but in addition two other pairs of "far" valence and conduction bands are introduced to order $k^{2}$. Since the effective masses for the conduction band $\left(m_{\|}^{\mathrm{cb}}=0.069 m_{0}\right.$ and $m_{\perp}^{\mathrm{cb}}=0.037 m_{0}$ at $\left.T=2 \mathrm{~K}\right)$ and for the valence band $\left(m_{\|}^{\mathrm{vb}}\right.$ $=0.066 m_{0}$ and $m_{\perp}^{\mathrm{vb}}=0.036 m_{0}$ at $T=2 \mathrm{~K}$ ) differ only slightly, we use instead of the complete Mitchell-Wallis band scheme, ${ }^{17}$ a simple two band model with mirrorlike symmetry of the conduction and valence bands. ${ }^{16-19}$ In this model a pair of interband matrix elements $P_{\|}$and $P_{\perp}$ describe the strong nonspherical anisotropy of the conduction and valence bands at the $L$ point of the Brillouin zone. The dispersion relations for the conduction and valence band energies are given by

$$
\begin{aligned}
E_{c}(k)= & \frac{\hbar^{2} k^{2}}{2 m_{0}}+\frac{1}{2 m_{0}}\left[4 \hbar^{2} P_{\perp}^{2}\left(k_{x}^{2}+k_{y}^{2}\right)+4 \hbar^{2} P_{\|}^{2} k_{z}^{2}\right. \\
& \left.+m_{0}^{2} E_{G}^{2}\right]^{1 / 2}, \\
E_{v}(k)= & \frac{\hbar^{2} k^{2}}{2 m_{0}}+\frac{1}{2 m_{0}}\left[4 \hbar^{2} P_{\perp}^{2}\left(k_{x}^{2}+k_{y}^{2}\right)+4 \hbar^{2} P_{\|}^{2} k_{z}^{2}\right. \\
& \left.+m_{0}^{2} E_{G}^{2}\right]^{1 / 2},
\end{aligned}
$$

where the zero of energy is taken at half the energy gap $E_{G}$. The $z$ axis is parallel to a [111] direction, i.e., perpendicular to the Brillouin-zone boundary at the $L$ point for the four constant energy ellipsoids, and $m_{0}$ is the free-electron mass. The interband momentum matrix elements $P_{\|}$and $P_{\perp}$ differ considerably for interband transitions parallel and perpendicular to the principal axes of the energy ellipsoid in $\mathbf{k}$ space. They are related to the anisotropic band-edge masses 


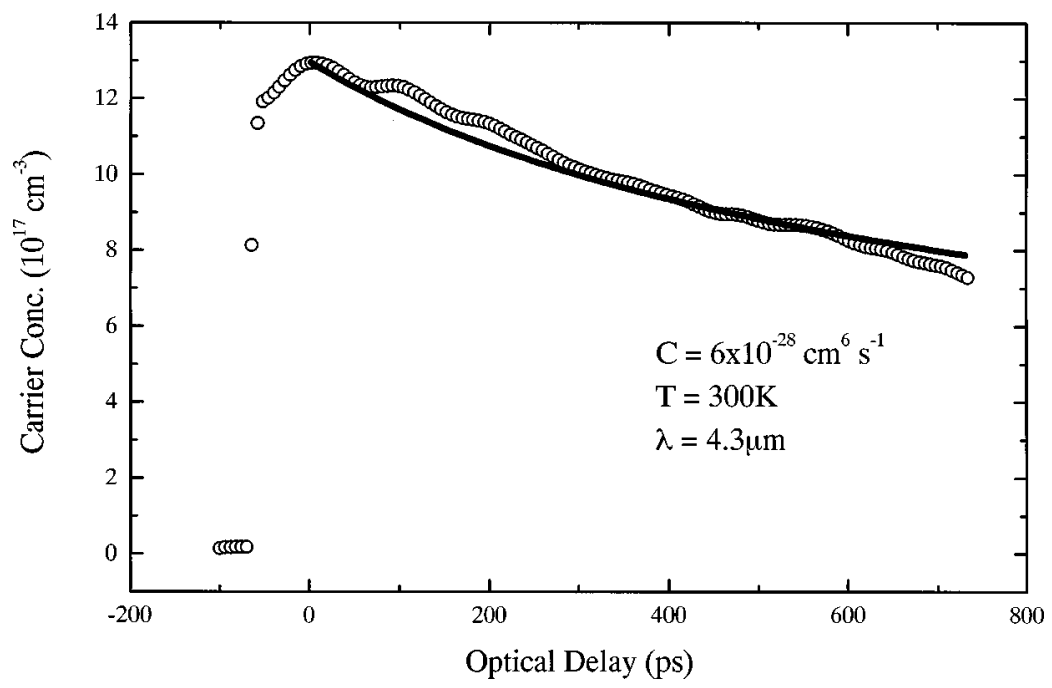

FIG. 5. Computed excited carrier concentration $N_{e}$ vs $t_{\text {delay }}$ obtained from the pump-probe transmission results as described in the text for $T=300 \mathrm{~K}$ and a pump wavelength $4.3 \mu \mathrm{m}$. The solid curve is the best fit from Eq. (9). The calculated value of $C$ is $6 \times 10^{-28} \mathrm{~cm}^{6} \mathrm{~s}^{-1}$.

$m_{\|}$and $m_{\perp}$ at the $L$ point of the Brillouin zone. ${ }^{17}$ Neglecting the far-band contributions, the effective two-band parameters are given by

$$
\frac{2 P_{\perp}^{2}}{m_{0}}=E_{G} \frac{m_{0}}{m_{\perp}}=4.01 \mathrm{eV}, \quad \frac{2 P_{\|}^{2}}{m_{0}}=E_{G} \frac{m_{0}}{m_{\|}}=2.17 \mathrm{eV},
$$

where the value $E_{G}=146.3 \mathrm{meV}$ was used. ${ }^{14}$ These values correspond to masses $m_{\|}=0.0675 m_{0}$ and $m_{\perp}=0.0365 m_{0}$ at $T=2 \mathrm{~K}$. With increasing temperature the masses become larger due to the increasing energy gap, and the energymomentum relationships [Eqs. (4) and (5)] change accordingly. In the two-band approximation we can use the following simple expression in Eq. (3) for the joint density of states: ${ }^{18}$

$$
J_{\mathrm{cv}}(E)=\frac{m_{0}^{3}}{8 \pi^{2} \hbar^{3} P_{\perp}^{2} P_{\|}} E\left(E^{2}-E_{G}^{2}\right)^{1 / 2}
$$

\section{RESULTS AND DISCUSSION}

We can now calculate the value of the excited carrier concentration, $N_{e}\left(t_{\text {delay }}\right)$, corresponding to each value of transmission, $T\left(t_{\text {delay }}\right)$. As described previously, we have also included the effect of small refractive index changes arising from a shift in the plasma frequency. ${ }^{13}$ Typical plots of $N_{e}$ versus $t_{\text {delay }}$ are shown in Figs. 5-7 for 300, 150, and $77 \mathrm{~K}$, respectively, showing clearly the rapid decay associated with stimulated emission at low temperatures which does not appear at room temperature at the excitation level of the present experiment. It has been shown previously ${ }^{6}$ that the carrier system of $\mathrm{PbSe}$ and $\mathrm{PbTe}$ becomes degenerate at the values of $N_{e}$ generated here for temperatures below 160 $\mathrm{K}$, with the quasi-Fermi levels shifted into the bands, giving the possibility of stimulated emission under the condition

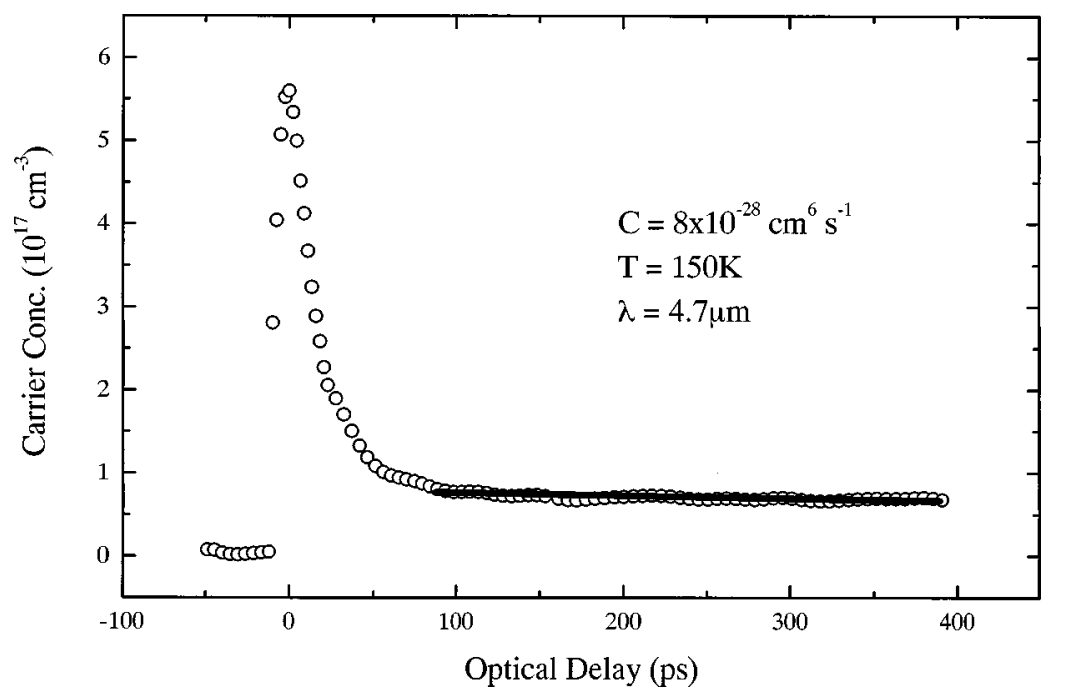

FIG. 6. Computed excited carrier concentration $N_{e}$ vs $t_{\text {delay }}$ obtained from the pump-probe transmission results as described in the text for $T=150 \mathrm{~K}$ and a pump wavelength $4.7 \mu \mathrm{m}$. The solid curve is the best fit from Eq. (9). The calculated value of $C$ is $8 \times 10^{-28} \mathrm{~cm}^{6} \mathrm{~s}^{-1}$. 


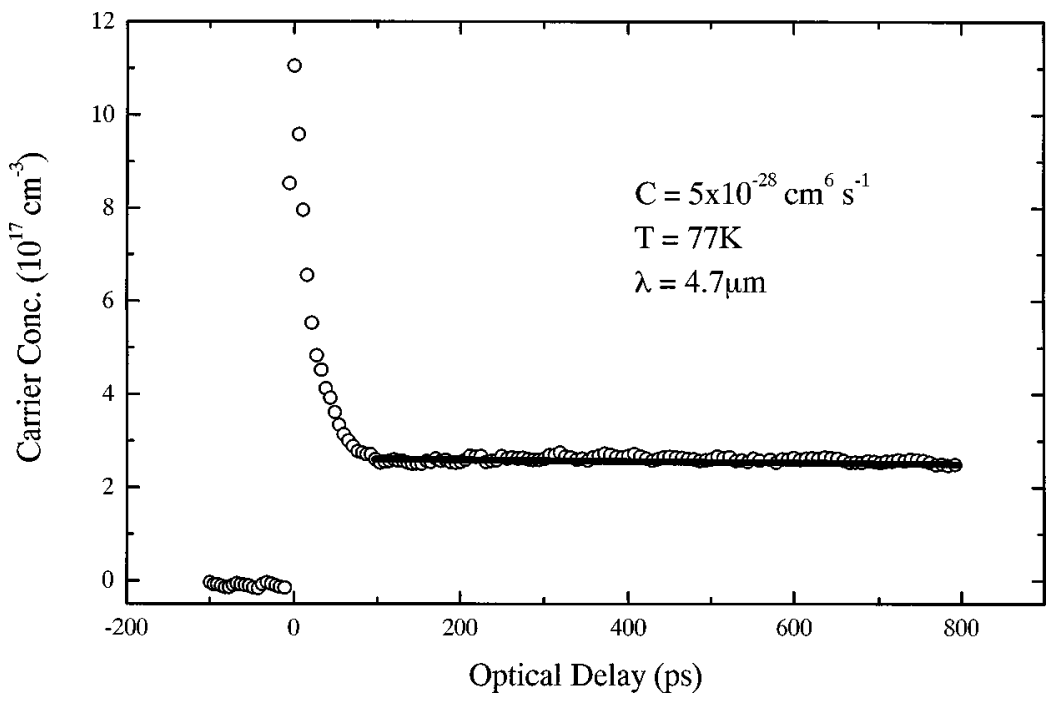

FIG. 7. Computed excited carrier concentration $N_{e}$ vs $t_{\text {delay }}$ obtained from the pump-probe transmission results as described in the text for $T=77 \mathrm{~K}$ and a pump wavelength $4.7 \mu \mathrm{m}$. The solid curve is the best fit from Eq. (9). The calculated value of $C$ is $5 \times 10^{-28} \mathrm{~cm}^{6} \mathrm{~s}^{-1}$.

The spontaneously emitted photons are amplified in the inverted sample, resulting in an exponential increase in photon number and a decrease of carrier density. Computed results for absorption and gain versus energy for different values of excited carrier concentrations are shown in Fig. 8. The stimulated emission reduces the carrier concentration very efficiently until it decreases below a threshold value defined by Eq. (8). The correlation of the onset of stimulated emission with the ps recombination kinetics shows that the carrier density in the excited volume is preferentially reduced by stimulated recombination at early delay times. From our observations of $N_{e}$ versus $t_{\text {delay }}$ plots at temperatures in the range 30-300 $\mathrm{K}$ we have determined the threshold value of $N_{e}$ as a function of temperature as shown in Fig. 9. Good agreement is obtained with the results of Ref. 6 at the two values previously reported, as shown in the figure, with values ranging from about $3 \times 10^{17} \mathrm{~cm}^{-3}$ at $70 \mathrm{~K}$ to 3 $\times 10^{18} \mathrm{~cm}^{-3}$ at $300 \mathrm{~K}$. The experimental threshold concentration at $300 \mathrm{~K}$ from our work is an estimated value obtained by changing the Fermi level until the absorption coefficient just becomes negative at the experimental pumping

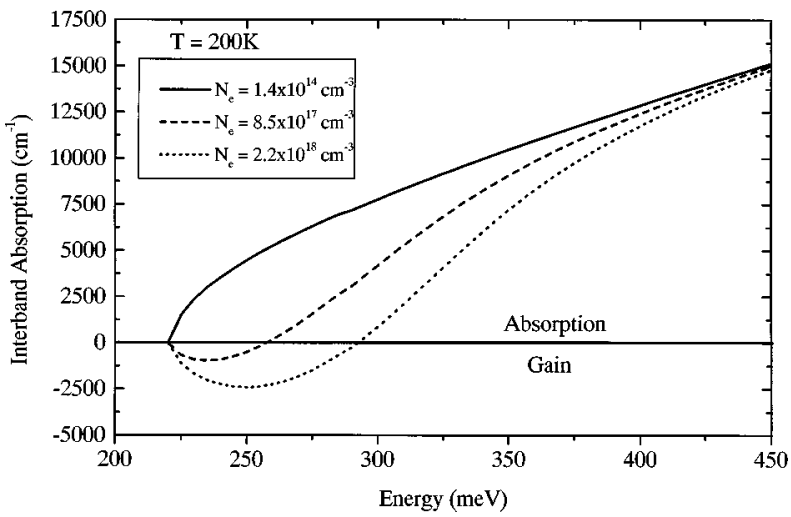

FIG. 8. Computed nonlinear interband absorption coefficient for $\mathrm{PbSe}$ for Eq. (3) as a function of energy for different values of excited carrier concentration at $T=200 \mathrm{~K}$. The transition from absorption to gain can be clearly seen for excited concentrations in excess of $\sim 10^{17} \mathrm{~cm}^{-3}$. wavelength, and calculating the corresponding carrier concentration.

For the data at room temperature, and for the results at lower temperatures in the regime below the threshold value of $N_{e}$ for stimulated emission, the data were analyzed with a simple rate equation for radiative and nonradiative decay, including terms depending on the Schockley-Reed, radiative, and Auger recombination coefficients $A, B$, and $C$, respectively, for a nondegenerate $N_{e}$ distribution:

$$
\frac{d N(t)}{d t}=A N(t)+B N^{2}(t)+C N^{3}(t)
$$

This equation was solved and fitted to the decay curves as shown in Figs. 5-7. It was found in all cases that Auger recombination dominated and the other terms were negligible, and in particular that the $N_{e}^{3}$ dependence expected for a nondegenerate carrier distribution gave a very good fit to the data. A similar result was obtained in the $300 \mathrm{~K}$ case in Ref. 6. The value of $C$ obtained by this fitting procedure is shown plotted over the full temperature range $30-300 \mathrm{~K}$ in Fig. 10. Excellent agreement between theory and experiment is obtained with the Auger process for intervalley scattering in the approximation of nondegenerate statistics, but it is clearly essential to include a realistic nonparabolic band structure. ${ }^{2}$ The difference between the parabolic and nonparabolic approximations is most clearly seen in the lowtemperature regime where the Auger coefficient drops markedly between 77 and $30 \mathrm{~K}$. Finally the Auger results for the lead salts are compared with earlier measurements taken at FELIX for $\mathrm{Hg}_{0.795} \mathrm{Cd}_{0.205} \mathrm{Te}$ (experiment and theory ${ }^{13,20,21}$ ) and $\mathrm{Hg}_{0.744} \mathrm{Cd}_{0.256} \mathrm{Te}$ (theory ${ }^{21}$ ). Since there is such extremely good agreement between the theory and nonparabolic bands and the isotropic Kane model, the latter curve (with the same band gap as PbSe at $100 \mathrm{~K}$-i.e., $\lambda_{G} \sim 7 \mu \mathrm{m}$ ) is taken for comparison between the two materials. It is found that $C$ for PbSe is between one and two orders of magnitude lower than for $\mathrm{Hg}_{1-x} \mathrm{Cd}_{x} \mathrm{Te}$ at comparable values of the band gap. Stimulated emission was not observed in the 


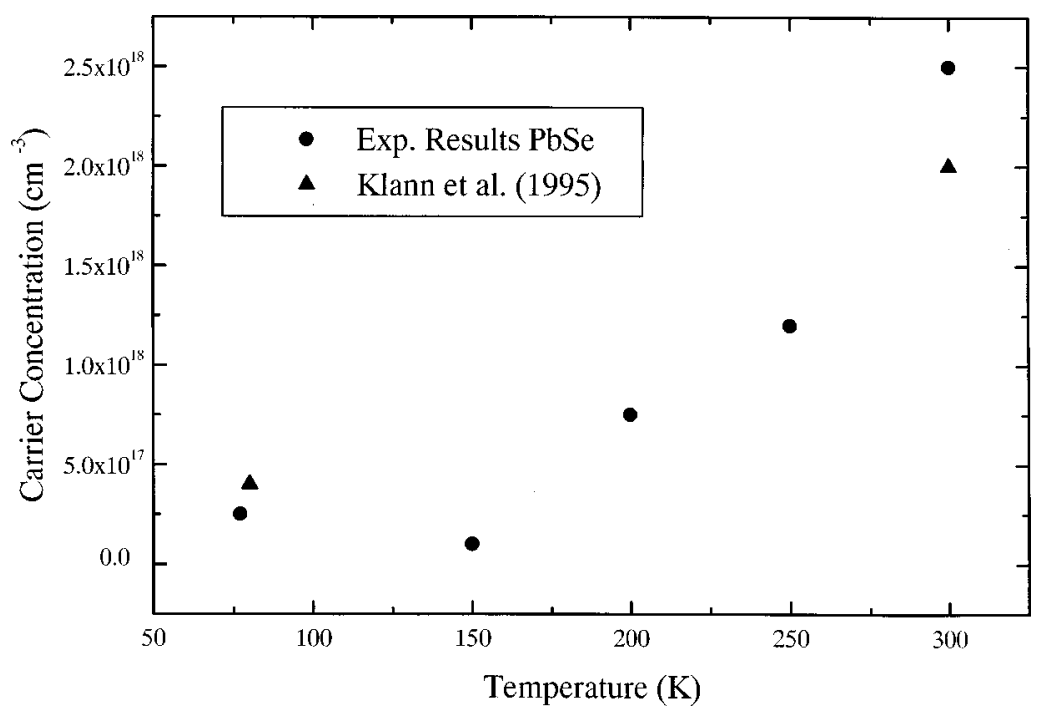

FIG. 9. Threshold excited concentration for simulated emission vs temperature for $n$-type PbSe (PS15)—circles, present work; triangles, Ref. 6.

earlier $\mathrm{Hg}_{0.795} \mathrm{Cd}_{0.205} \mathrm{Te}$ measurements even at the lowest temperatures used, but it is possible that it might be achieved in material of precisely the same bandgap as PbSe (i.e., $x$ $=0.256$ ) and with the higher FELIX intensities available with the current optical setup. We estimate the threshold carrier concentration for this material would be about 8 $\times 10^{17} \mathrm{~cm}^{-3}$, which is some four times greater than achieved in the earlier experiments. ${ }^{13,20}$

One other clear difference between the two systems is in the dependence of $C$ on temperature. Both $\mathrm{PbSe}$ and $\mathrm{Hg}_{1-x} \mathrm{Cd}_{x} \mathrm{Te}$ (of this composition) are anomalous in that the band gap decreases with lowering temperature. However, in the case of $\mathrm{Hg}_{1-x} \mathrm{Cd}_{x} \mathrm{Te}$, because of the low ratio of electron to heavy-hole effective mass arising from the Kane band structure, which decreases further with decreasing band gap, the threshold for Auger recombination drops with reducing temperature. This band gap dependence dominates over the change of the occupation function with temperature. By contrast, the ratio of electron to hole effective mass for $\mathrm{PbSe}$ is approximately unity over the whole range of values of $E_{G}$. In this case reducing the temperature below threshold quenches the Auger recombination.

\section{CONCLUSION}

In summary, we have utilized a ps free-electron laser to measure directly the Auger recombination rates as a function of photonexcited carrier concentration in PbSe over a wide temperature range $(30-300 \mathrm{~K})$. The rate is approximately constant between 77 and $300 \mathrm{~K}$, with a value $C=8$ $\times 10^{-28} \mathrm{~cm}^{6} \mathrm{~s}^{-1}$, and then drops to a value of $C=1$ $\times 10^{-28} \mathrm{~cm}^{6} \mathrm{~s}^{-1}$ at $30 \mathrm{~K}$. Good agreement is obtained with

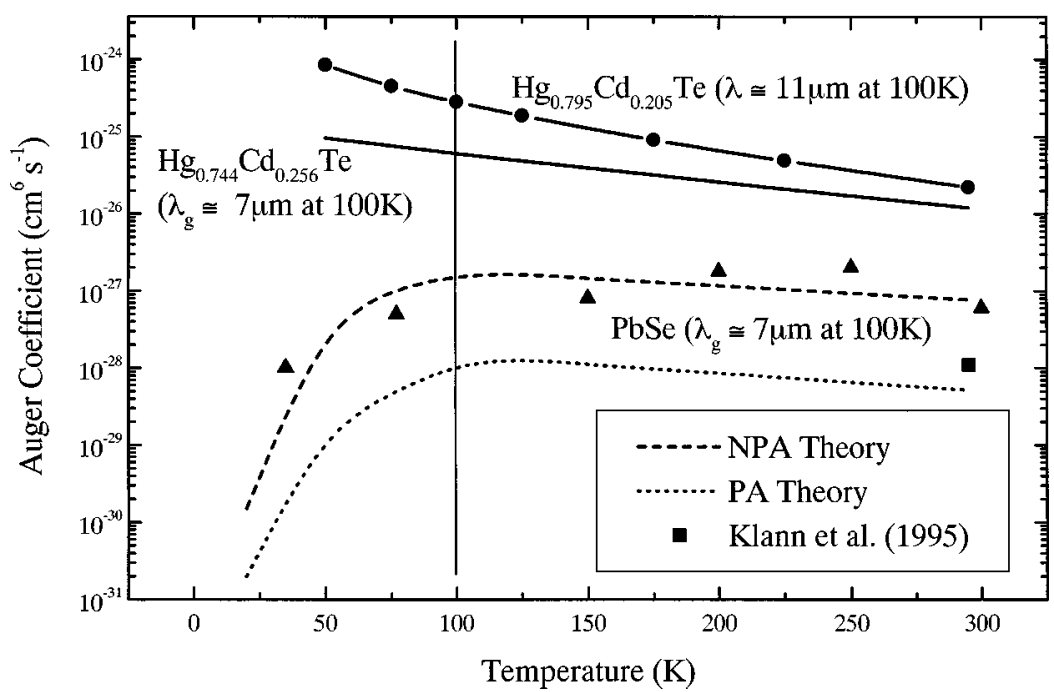

FIG. 10. PbSe: Auger coefficient $C$ vs temperature for $n$-type PbSe (PS15)—solid triangles, present work; solid square, Ref. 6. The dashed line is the theoretical curve for a nondegenerate distribution and nonparabolic bands (NPA) after Ref. 2; the dotted line is from the same paper in the parabolic band (PA) approximation. $\mathrm{Hg}_{1-x} \mathrm{Cd}_{x} \mathrm{Te}$ : Auger coefficient $C$ vs temperature for $\mathrm{Hg}_{0.795} \mathrm{Cd}_{0.205} \mathrm{Te}-\mathrm{circles}$ The solid lines are the theoretical curves for two compositions $\left(\mathrm{Hg}_{0.795} \mathrm{Cd}_{0.205} \mathrm{Te}\right.$ and $\left.\mathrm{Hg}_{0.744} \mathrm{Cd}_{0.256} \mathrm{Te}\right)$ for the Kane model of nonparabolic, spherical bands (Refs. 13, 20, and 21). The energy gap for PbSe and $\mathrm{Hg}_{0.744} \mathrm{Cd}_{0.256} \mathrm{Te}$ is the same $(\sim 7 \mu \mathrm{m})$ at $T=100 \mathrm{~K}$. 
theoretical calculations for nonparabolic, nonspherical energy bands in the non-degenerate limit for the excited carrier concentration. ${ }^{2}$ Good agreement is also obtained with the room-temperature value for $C$ reported previously, and we confirm the earlier result that for temperatures below $200 \mathrm{~K}$ and carrier densities above the threshold for stimulated emission, stimulated recombination represents the most efficient recombination mechanism. ${ }^{6}$ We have determined the threshold carrier concentration for stimulated emission over the same temperature range, 30-300 K, and this occurs at carrier concentrations somewhat lower than would be the case for bulk materials with the Kane band structure. This is in accord with the finding that $C$ for $\mathrm{PbSe}$ is between one and two orders of magnitude lower than for InSb or $\mathrm{Hg}_{1-x} \mathrm{Cd}_{x} \mathrm{Te}$ of comparable values of the band gap. The small Auger coefficients in the lead salts are mitigated to some extent by the relatively heavy anisotropic effective masses and valley degeneracy, which would tend, by contrast, to higher threshold carrier concentrations than for $\mathrm{Hg}_{1-x} \mathrm{Cd}_{x} \mathrm{Te}$ or III-V materi- als of comparable band gaps (assuming comparable internal losses).

We confirm the conjecture made recently that Auger recombination in IV-VI semiconductors in the wavelength range of 3-5 $\mu \mathrm{m}$ will not prevent quantum structures of these materials from achieving room-temperature laser operation. ${ }^{6}$ Rather, it is the problems of insufficient electron and hole confinement and low thermal conductivity from traditionally used materials, $\mathrm{PbEuSeTe}, \mathrm{PbSrSe}$, or $\mathrm{PbSrS}$, that have to be overcome by the utilization of structures such as $\mathrm{GaSb} / \mathrm{PbSe} / \mathrm{GaSb}{ }^{5}$

\section{ACKNOWLEDGMENTS}

We acknowledge the EPSRC for the support of this work. We are grateful to DERA Malvern for financial support (C.M.C. and P.C.F.). Two of us (C.M.C. and P.C.F.) were supported by the EPSRC. The work was also supported by the Osterreichischer Fonds zur Förderung der Wissenschaftlicken Forschung.
${ }^{1}$ P. R. Emtage, J. Appl. Phys. 47, 2565 (1976).

${ }^{2}$ O. Ziep, O. Mocker, D. Genzow, and K. H. Hermann, Phys. Status Solidi B 90, 197 (1978).

${ }^{3}$ M. Mocker and O. Ziep, Phys. Status Solidi B 115, 415 (1983).

${ }^{4}$ R. Rosman and A. Katzir, IEEE J. Quantum Electron. QE-18, 814 (1982).

${ }^{5}$ Z. Shi, Appl. Phys. Lett. 72, 1272 (1998).

${ }^{6}$ R. Klann, T. Hofer, R. Buhleir, T. Elsaesser, and J. W. Tomm, J. Appl. Phys. 77, 277 (1995); J. W. Tomm, M. Mocker, T. Kelz, T. Elsaesser, R. Klann, B. V. Novikov, V. G. Talalaev, V. E. Tudorovskii, and H. Bottner, J. Appl. Phys. 78, 7247 (1995).

${ }^{7}$ K. Lischka and W. Huber, J. Appl. Phys. 48, 2632 (1977).

${ }^{8}$ T. X. Hoai and K. H. Herrmann, Phys. Status Solidi B 83, 465 (1977)

${ }^{9}$ R. Klann, R. Buhleier, T. Elseasser, and A. Lambrecht, Appl. Phys. Lett. 59, 885 (1991).

${ }^{10}$ R. Klann, T. Hofer, R. Buhleier, T. Elsaesser, and A. Lambrecht, Appl. Phys. Lett. 61, 2866 (1992).
${ }^{11}$ D. L. Partin, IEEE J. Quantum Electron. QE-24, 1716 (1988).

${ }^{12}$ M. Tacke, Infrared Phys. Technol. 36, 447 (1995).

${ }^{13}$ C. M. Ciesla, B. N. Murdin, T. J. Phillips, A. M. White, A. R. Beattie, C. J. G. M. Langerak, C. T. Elliott, C. R. Pidgeon, and S. Sivananthan, Appl. Phys. Lett. 71, 491 (1997).

${ }^{14}$ G. Springholz and G. Bauer, Appl. Phys. Lett. 60, 1600 (1992).

${ }^{15}$ C. M. Ciesla, B. N. Murdin, C. R. Pidgeon, R. A. Stradling, C. C. Phillips, M. Livingstone, I. Gabraith, D. A. Jaroszynski, C. J. G. M. Langerak, P. J. P. Tang, and M. J. Pullin, J. Appl. Phys. 80, 2994 (1996).

${ }^{16}$ J. O. Dimmock and G. B. Wright, Phys. Rev. A 135, 821 (1964).

${ }^{17}$ D. L. Mitchell and R. F. Wallis, Phys. Rev. 151, 581 (1966).

${ }^{18}$ S. Yuan, H. Krenn, G. Springholz, and G. Bauer, Phys. Rev. B 47, 7213 (1993).

${ }^{19}$ H. Pascher, G. Bauer, and R. Grisar, Phys. Rev. B 38, 3383 (1988).

${ }^{20}$ C. M. Ciesla, Ph.D. thesis, Heriot Watt, 1997 (unpublished).

${ }^{21}$ A. R. Beattie and A. M. White, J. Appl. Phys. 79, 802 (1996). 